\section{Effects of spontaneous breathing activity on regional and global lung inflammation at different PEEP levels in experimental moderate ARDS}

\author{
J. Wittenstein, T. Kiss, T. Bluth, A. Braune, R. Huhle, M. Gama de Abreu
}

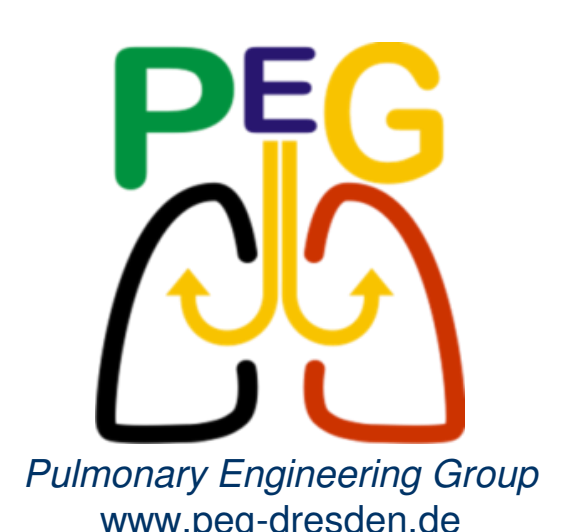

Puw.peg-dresden.de

Department of Anaesthesiology and Intensive Care Medicine, University Hospital Carl Gustav Carus, Dresden, Germany

\section{Background}

Spontaneous breathing activity (SB) in acute respiratory distress syndrome (ARDS) can further aggravate the lung injury. Positive end-expiratory pressure (PEEP) has the potential to stabilize the lungs and prevent damage. We hypothesized that in experimental moderate ARDS SB combined with inadequate low PEEP increases inflammation in dependent lung areas.

\section{Methods}

24 pigs were anesthetized, and lung injury was induced with multiple saline lung lavage followed by injurious ventilation (double-hit modell) (Fig. 1).

- For assessment of transpulmonary pressure $\mathrm{P}_{\mathrm{TP}}$ intrapleural pressure sensors were placed in ventral, dorsal and caudal regions of the left hemithorax (Fig. 2).

- Animals were ventilated with low tidal volumes $\left(V_{t}=6 \mathrm{ml} / \mathrm{kg}\right)$ in airway pressure release ventilation (APRV) mode.

- Best PEEP (PEEPb) was defined as the level needed to reach a positive end-expiratory transpulmonary pressure in all lung regions.

- Animals were randomized to one of four groups ( $n=6 /$ group):

- 1) no SB activity and PEEP=PEEPb-4 $\mathrm{cmH}_{2} \mathrm{O}$ (APRV/Clow);

- 2) no SB activity and PEEP=PEEPb+4 $\mathrm{cmH}_{2} \mathrm{O}$ (APRV/Chigh);

- 3) SB activity and PEEP=PEEPb-4 $\mathrm{cmH}_{2} \mathrm{O}$ (APRV/SBlow);

- 4) SB activity and PEEP=PEEPb+4 $\mathrm{cmH}_{2} \mathrm{O}$ (APRV/SBhigh).

Regional lung $\left[{ }^{18} \mathrm{~F}\right]$ fluorodeoxyglucose $\left(\left[{ }^{18} \mathrm{~F}\right] \mathrm{FDG}\right)$ uptake as well as perfusion using ${ }^{68} \mathrm{Ga}$-labeled microspheres were assessed by positron emission tomography (PET) after 12 hours.

- The specific $\left[{ }^{18} \mathrm{~F}\right] \mathrm{FDG}$ uptake rate $\left(\mathrm{K}_{\text {is }}\right)$ was calculated and lung aeration determined by static computed tomography.

\section{Results}

- At low PEEP, global $K_{\text {is }}$ increased both with and without $S B$ activity compared with high PEEP (Fig 3, 4).

- SB activity did not increase global lung inflammation, regardless of PEEP level (Fig. 4).

- Nevertheless, transpulmonary driving pressure resulting from SB activity correlated with regional $\mathrm{K}_{\text {is }}$ at low but not high PEEP (Fig. 5).

- In addition, the distribution of regional transpulmonary pressures at end-expiration across the gravity gradient was more homogeneous with high PEEP (Fig. 5).

- The centers of aeration and perfusion were shifted towards dependent lung regions in APRV/SBhigh compared with APRV/SBlow (Fig. 3).

\section{Conclusion}

In this model of moderate ARDS in pigs, SB activity at inadequate low levels of PEEP correlates with dorsal lung inflammation.

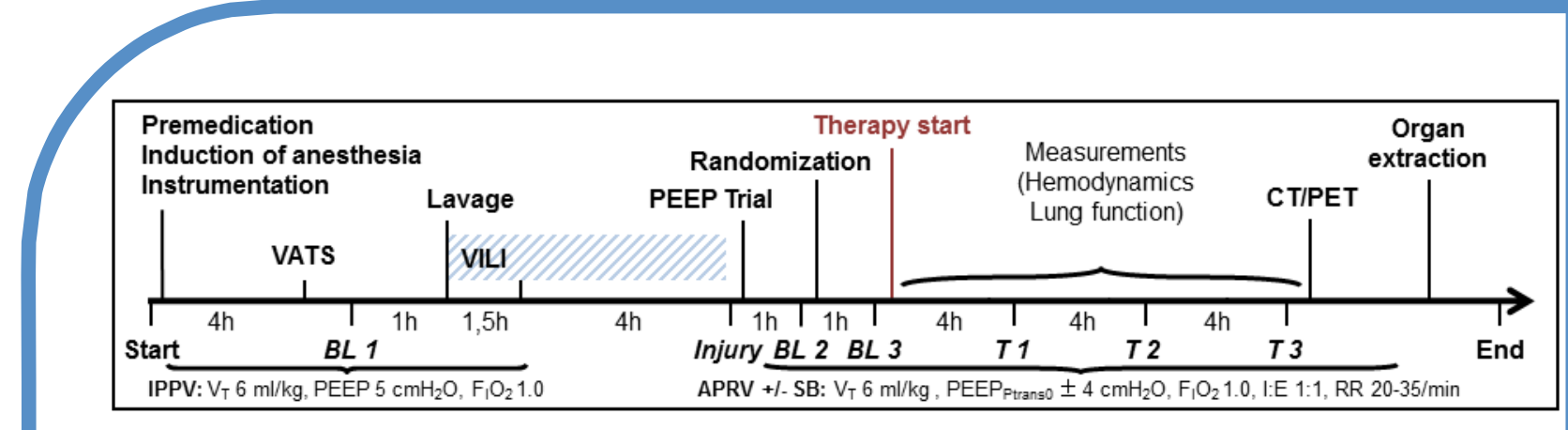

Fig. 1: Time course of interventions and measurements.
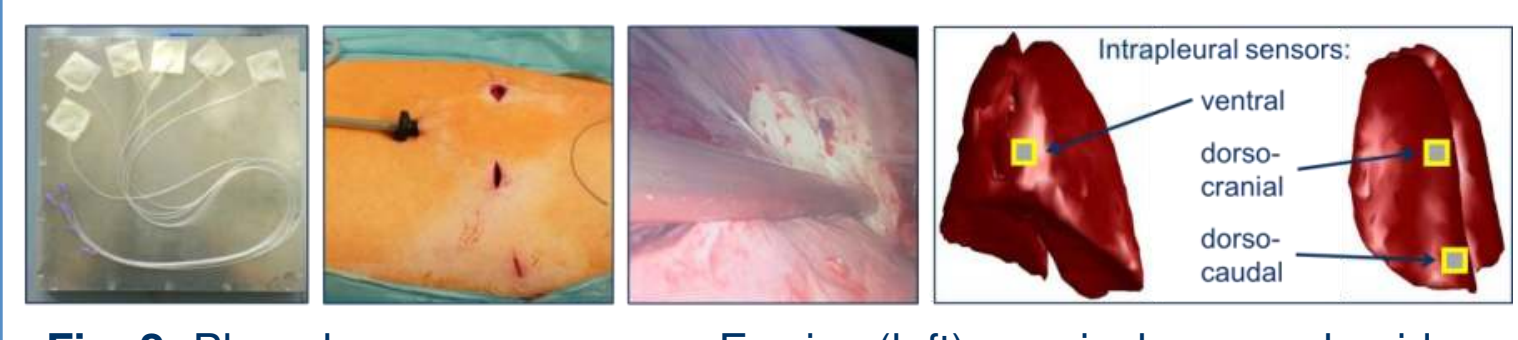

Fig. 2: Pleural pressure sensors. Ex vivo (left), surgical approach; video thoracoscopy and scheme of positioning (right).

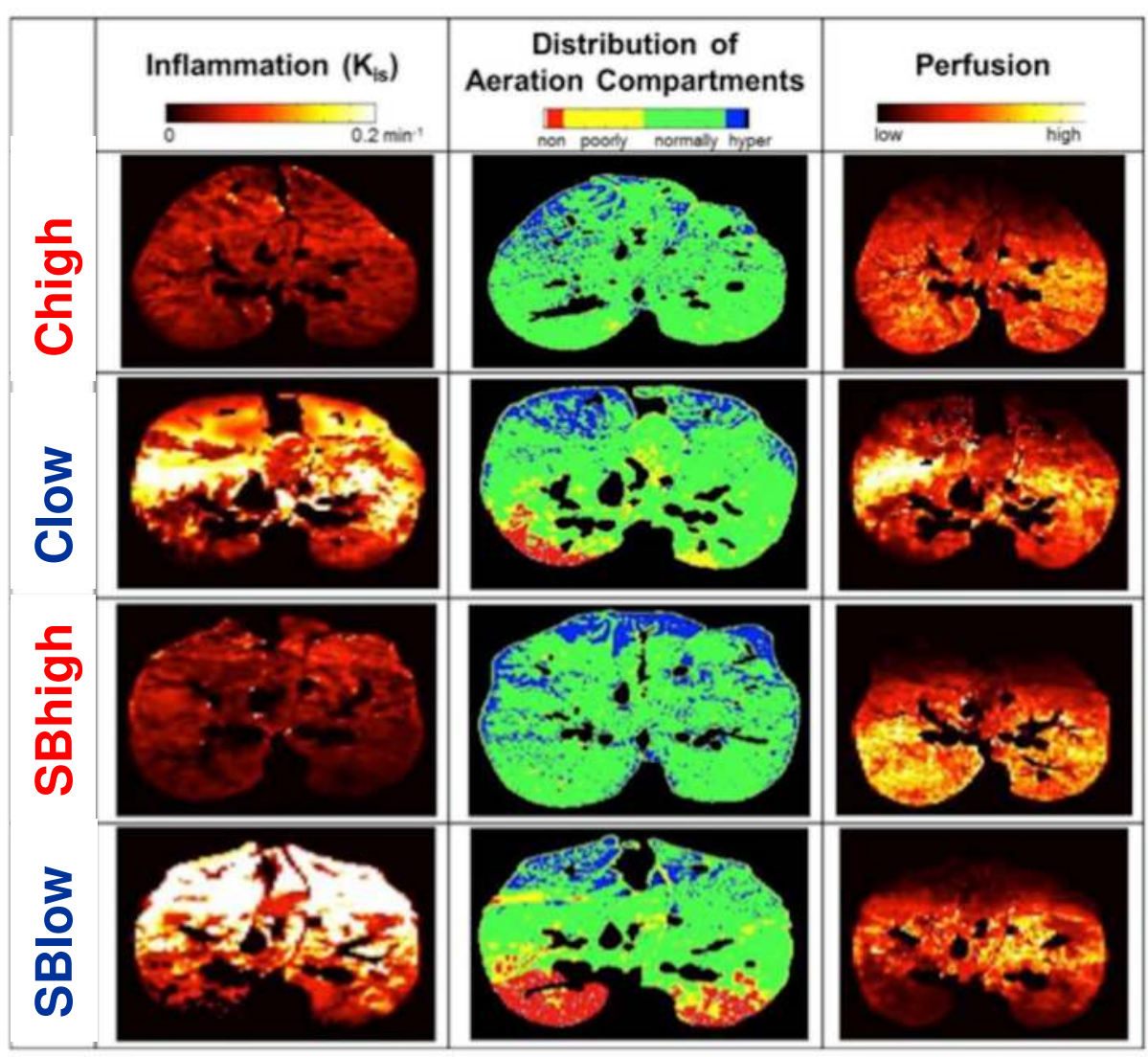

Fig. 3: Single slice images of distribution of lung inflammation $\left(\mathrm{K}_{\mathrm{iS}}\right)$, aeration compartments from computer tomography and perfusion from positron emission tomography in one representative animal of each group.

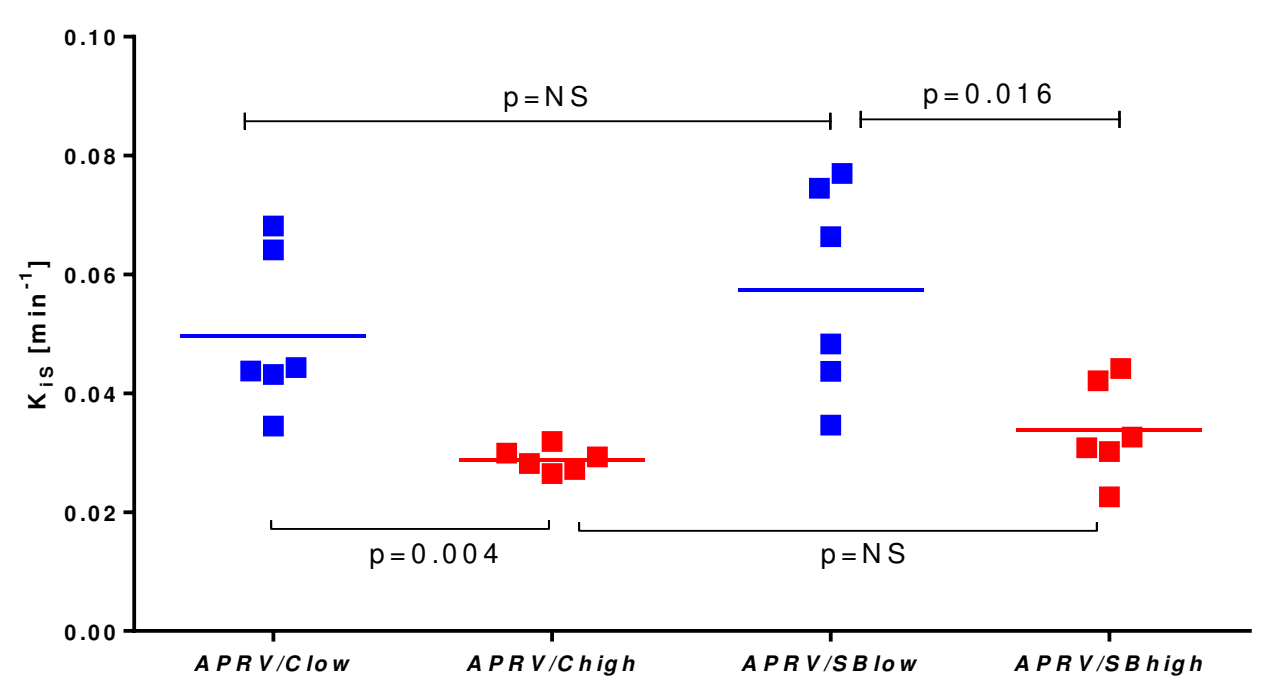

Fig. 4: Global lung ${ }^{18} \mathrm{~F}-\mathrm{FDG}$ uptake rate $\left(\mathrm{K}_{\mathrm{i}}\right)$ normalized to fractional tissue content $\left(K_{i S}=K_{i} /\left[1-F_{\text {blood }}-F_{\text {gas }}\right]\right)$. Mean. Mann Whitney test.

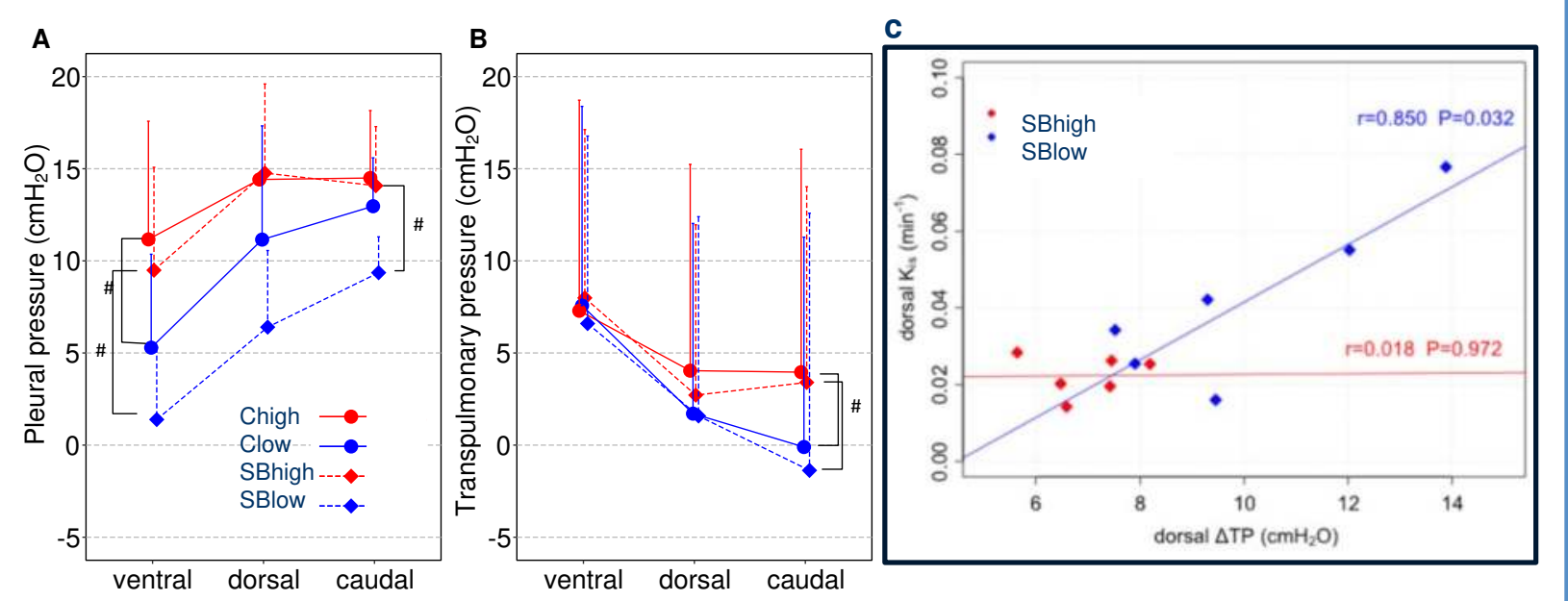

Fig. 5: A) End-expiratory pleura pressure. Mean and SD, $\# p<0,05$; B) Endexpiratory transpulmonary pressure. Mean and SD, \#p<0,05; C) Association between inflammation and $\Delta$ transpulmonary pressure $(\Delta T P)$ in dorsal lung regions. 